\title{
Osmotic stress and cryoinjury of koala sperm: an integrative study of the plasma membrane, chromatin stability and mitochondrial function
}

\author{
S D Johnston, N Satake, Y Zee, C López-Fernández ${ }^{1}$, W V Holt ${ }^{2}$ and J Gosálvez ${ }^{1}$ \\ School of Agriculture and Food Science, The University of Queensland, Gatton, Queensland 4343, Australia, \\ ${ }^{1}$ BioEdificio de Biología, Universidad Autónoma de Madrid, Cantoblanco, Madrid 28049, Spain and \\ ${ }^{2}$ Institute of Zoology, Zoological Society of London, Regent's Park, London NW1 4RY, UK \\ Correspondence should be addressed to WV Holt; Email: bill.holt@ioz.ac.uk
}

\begin{abstract}
This study investigated whether cryopreservation-induced injury to koala spermatozoa could be explained using an experimental model that mimics the structural and physiological effects of osmotic flux. DNA labelling after in situ nick translation of thawed cryopreserved spermatozoa revealed a positive correlation $(r=0.573 ; P<0.001 ; n=50)$ between the area of relaxed chromatin in the nucleus and the degree of nucleotide labelling. While the chromatin of some spermatozoa increased more than eight times its normal size, not all sperm nuclei with relaxed chromatin showed evidence of nucleotide incorporation. Preferential staining associated with sperm DNA fragmentation (SDF) was typically located in the peri-acrosomal and peripheral regions of the sperm head and at the base of the spermatozoa where it appear to be 'hot spots' of DNA damage following cryopreservation. Results of the comparative effects of anisotonic media and cryopreservation on the integrity of koala spermatozoa revealed that injury induced by exposure to osmotic flux, essentially imitated the results found following cryopreservation. Plasma membrane integrity, chromatin relaxation and SDF appeared particularly susceptible to extreme hypotonic environments. Mitochondrial membrane potential (MMP), while susceptible to extreme hypo- and hypertonic environments, showed an ability to rebound from hypertonic stress when returned to isotonic conditions. Koala spermatozoa exposed to $64 \mathrm{mOsm} / \mathrm{kg}$ media showed an equivalent, or more severe, degree of structural and physiological injury to that of frozen-thawed spermatozoa, supporting the hypothesis that cryoinjury is principally associated with a hypo-osmotic effect. A direct comparison of SDF of thawed cryopreserved spermatozoa and those exposed to a $64 \mathrm{mOsm} / \mathrm{kg}$ excursion showed a significant correlation $(r=0.878 ; P<0.05 ; n=5)$; however, no correlation was found when the percentage of sperm with relaxed chromatin was compared. While a cryo-induced osmotic injury model appears to explain post-thaw changes in koala SDF, the mechanisms resulting in relaxed chromatin require further study. A lack of correlation between the percentage of sperm with relaxed chromatin and SDF suggests that the timing of these pathologies are asynchronous. We propose an integrative model of cryo-induced osmotic injury that involves a combination of structural damage (rupture of membrane) and oxidative stress that first leads to the reduction of MMP and the relaxation of chromatin, which is then ultimately followed by an increase in DNA fragmentation.
\end{abstract}

Reproduction (2012) 143 787-797

\section{Introduction}

The cryopreservation process exposes spermatozoa to an extreme range of fluctuating osmotic conditions. These conditions exert physical and biochemical stress on spermatozoa that influences their ability to survive the freeze-thaw process (Watson \& Fuller 2001). Extracellular ice crystals formed during sperm cryopreservation result in an increase in solute concentration within unfrozen pockets of liquid and consequently form hypertonic environments so that subsequent equilibration of the solute concentration between intraand extracellular environments results in osmotic efflux of water across cellular membranes. This in turn leads to a reduction of cellular volume and a distortion of the molecular and cytoplasmic organisation of organelles. During thawing, and removal of the cryoprotective agent, the osmotic environment is restored, and an influx of water across the sperm plasma membrane causes further damage to the already compromised cell (Curry \& Watson 1994). Species-specific structural and physiological parameters inherent to the spermatozoa determine their capacity to respond to changes in temperature and osmolality during cryopreservation; particularly important are differences in plasma membrane composition that can affect membrane 
permeability to water, ions and solutes and the lipid phase transition temperature (Holt 2000). By contrast, the effect of cryopreservation on DNA structure and stability has received remarkably less attention.

The majority of marsupial spermatozoa have no cysteine residues in their protamines (Retief et al. 1995) and consequently lack the stabilising disulphide bonds normally found in the sperm head of eutherian mammals; consequently, marsupial spermatozoa have been shown to be particularly susceptible to damage during air-drying or exposure to high divalent salt concentrations and detergents (Cummins 1980). The koala (Phascolarctos cinereus) is one species in which the effects of cryopreservation have been investigated (Johnston et al. 1994, 2006, Zee et al. 2007, 2008, 2009a). For example, koala spermatozoa have been shown to have a post-thaw viability of up to $60-70 \%$ if frozen in high levels of glycerol $(14 \% \mathrm{v} / \mathrm{v})$ combined with a relatively slow freezing rate of $6^{\circ} \mathrm{C} / \mathrm{min}$. However, extended incubation of thawed koala spermatozoa for a further $2 \mathrm{~h}$ rapidly reduces the percentage of live spermatozoa and results in a corresponding increase in the number of cells with swollen or relaxed chromatin (Zee et al. 2008, 2009b). It is uncertain whether this modified form of chromatin is either a consequence of physical swelling or distortion of the DNA/protamine complex in response to osmotically induced injury or is associated with an increase in the fragmentation of the DNA molecule (Johnston et al. 2007, Zee et al. 2009b).

Mitochondrial membrane potential (MMP) has been shown to be a sensitive indicator of the bioenergetic functions within the mitochondrion (Duchen 2004), and while Zee et al. (2007, 2009a, 2009b) have previously used the JC1 probe $\left(5,5^{\prime}, 6,6^{\prime}\right.$-tetrachloro$1,1^{\prime}, 3,3^{\prime}$-tetraethylbenzimidazolyl carbocyanine iodide) to investigate the effect of cryoinjury on the koala sperm midpiece, the relative detrimental effects of osmotic stress vs structural damage associated with intracellular ice crystal damage remain unresolved. While the response of spermatozoa to the damaging effects of osmotic stress during cryopreservation can be demonstrated artificially by exposure of spermatozoa to a range of osmotically defined media (Curry \& Watson 1994, Meyers 2005, Willoughby et al. 1996), to our knowledge, this experimental model has never been used before to examine the effect of osmosis on sperm DNA, and certainly not for marsupial sperm chromatin. In fact, the aim of this investigation was to examine whether osmotic flux is one of the primary causes of koala sperm cryoinjury. The first objective was to investigate the phenomenon of koala sperm chromatin relaxation postcryopreservation associated with a loss of membrane integrity (Johnston et al. 2006, Zee et al. 2008, 2009a) and to determine whether it was linked to a corresponding increase in sperm DNA fragmentation (SDF). Our second objective was to examine the effect of varying anisosmotic media on the MMP of koala spermatozoa and directly compare the observations of spermatozoa from the same ejaculate following cryopreservation. This study was designed to differentiate the integrative effects of cryoinjury on koala spermatozoa that could be attributed to osmotic flux.

\section{Results}

\section{Sperm chromatin relaxation}

Although the koala sperm nucleus can display a high degree of plesiomorphy, the most common shape is one of a 'hooked' or 'sickle' appearance (Fig. 1a). Upon post-thaw incubation at $35{ }^{\circ} \mathrm{C}$ for $30 \mathrm{~min}, \sim 14 \%$ of the koala spermatozoa demonstrated a massive increase in the size of the sperm head associated with a swelling or an apparent decondensation of the DNA-protamine complex (Fig. 1a, b, c and e). Spermatozoa with an
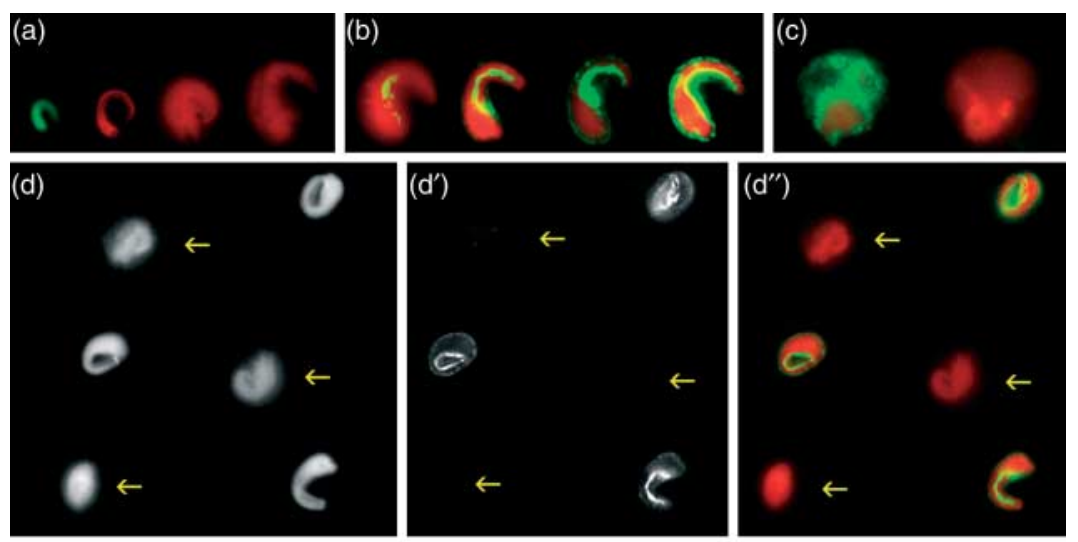

(e)

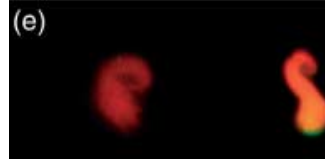

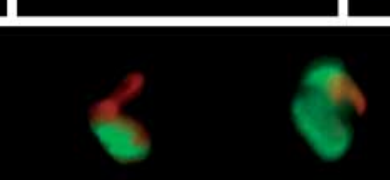

Reproduction (2012) 143 787-797
Figure 1 Koala sperm nuclei: (a) range of sperm nuclei showing relaxed chromatin - note small-sized green (SYBR-14) intact nucleus on the left side and the progressively larger red $(\mathrm{PI}+)$ membrane damaged nuclei on the right side; (b) sperm nuclei labelled with ISNT but no protein lysis - note nucleotide staining in the peri-acrosomal and nuclear periphery; (c) sperm nuclei with highly relaxed chromatin without protein lysis - nucleus on left side has ISNT staining, whereas the nucleus on the right side shows no evidence of DNA damage; (d) sperm nuclei stained with propidium iodide $(\mathrm{PI}) ;\left(\mathrm{d}^{\prime}\right)$ same sperm nuclei stained with ISNT - note partially relaxed nuclei with no evidence of DNA fragmentation (yellow arrow); ( $\left.d^{\prime \prime}\right)$ same sperm nuclei with ISNT and PI background staining; (e) sperm nuclei with variable degrees of chromatin relaxation and ISNT staining - note nucleotide staining commencing at the base of the head and the large nucleus with relaxed chromatin on right with no evidence of fragmented DNA. 
expanded nucleus were defined in this study as possessing a fluorescent signal at least double in area (measured in number of pixels) to that of a spermatozoon with an intact plasma membrane, although in some cases the nucleus expanded up to eight times its original size (Fig. 1a).

\section{Sperm DNA fragmentation}

SDF was assessed in this experiment using two different methodologies: the sperm chromatin dispersion (SCD) test and direct labelling of putative DNA breaks by in situ DNA extension using DNA polymerase I endonuclease free. After application of the SCD test, sperm nuclei were classified as either non-fragmented (2a: white arrows) or fragmented (2a: yellow and red arrows). Nonfragmented sperm nuclei showed no evidence of chromatin dispersion and in some instances maintained the original shape of the sperm head (Fig. 2a and b); fragmented sperm nuclei displayed a continuum of chromatin fragmentation from a small-to-medium (Fig. 2a and c, yellow arrows)-sized halo of chromatin dispersion to a massive chromatin dispersion of fragmented DNA and a corresponding decrease in size of the nuclear core (Fig. 2a, red arrow, d). It should be noted that chromatin dispersion in this context refers to the movement of the damaged sperm DNA in the SCD microgel following protein lysis. This phenomenon is different to that of chromatin relaxation, which is akin to a form of chromatin unwinding or swelling that is not necessarily associated with fragmented DNA. Spermatozoa identified as presenting with fragmented DNA were strongly labelled following the SCD and in situ nick translation (ISNT) procedures (Fig. 2c and d); the high activity of the DNA polymerase in this case was due to the existence of accessible $5^{\prime} / 3^{\prime}$ ends after protein removal, leading to the production of an intensely labelled halo. The spermatozoa that were not affected by the protein depletion remained unlabelled (Fig. 2b).

\section{Mapping sperm DNA damage}

Although the highly compacted nature of the chromatin prevents in some instances the full accessibility of the polymerase for labelling the DNA breaks, ISNT performed directly on koala sperm nuclei without protein depletion was still capable of targeting sperm with DNA breakage. We observed that the ISNTmodified nucleotides were not randomly incorporated in the nucleus but were primarily confined to two specific locations: a peri-acrosomal (Fig. 1b, d, d $d^{\prime}, d^{\prime \prime}$ ) and a basal region (Fig. 1e). While there was a general trend for the ability of the polymerase to increase the level of incorporated labelled nucleotides as the size of the chromatin increased or relaxed (compare Fig. $1 \mathrm{~b}$ with $c$ and different sized spermatozoa in Fig. 1e), there


Figure 2 Sperm DNA fragmentation of koala spermatozoa: (a) non-fragmented (white arrows) and fragmented koala sperm nuclei (small to medium - yellow arrows and large - red arrow); (b, c and d) ISNT following protein lysis - (b) non-fragmented; (c) small to medium halo fragmented and (d) large halo fragmented with highly dispersed chromatin. 
were also spermatozoa that showed a high degree of chromatin relaxation but remained unlabelled (Fig. 1c and arrow in $\left.d-d^{\prime \prime}\right)$. Conversely, there were other sperm nuclei showing no evidence of massive chromatin relaxation that were labelled by ISNT (Fig. $1 \mathrm{~d}-\mathrm{d}^{\prime \prime}$ ).

To assess a possible relationship between the degree of chromatin relaxation and the extent of DNA damage, a Pearson's $r$ correlation analysis was conducted. Figure 3 depicts the relationship between the area of the sperm chromatin (red fluorescence, measured in pixels as visualised in Fig. 1b, relaxed chromatin) and the incidence of DNA ISNT label (green fluorescence as visualised in Fig. 1b, SDF) for 50 thawed incubated spermatozoa. There was positive correlation $(r=0.573$; $P<0.001)$ between the degree of chromatin relaxation and the extent of DNA labelling following ISNT. It is interesting to note that the primary regional sperm mapping of DNA labelling commenced on the chromatin around the acrosomal region but expanded from this region to the whole periphery of the spermatozoon (Fig. $1 \mathrm{~b}$ and $\mathrm{d}$ ). The selective incorporation of nucleotides to the basal region of the spermatozoon was also observed in some cells (Fig. 1e). In these latter nuclei, the expansion of the DNA labelling occurred from the base of the sperm head to the distal extremity (Fig. 1e), and there was also a tendency towards increased DNA labelling as the chromatin became more relaxed; note, however, that



Figure 3 Correlation between chromatin area measured in pixels (red fluorescence) and the integrated density of the DNA ISNT label (green fluorescence) for 50 thawed incubated spermatozoa from two koalas. in other sperm nuclei, the highly relaxed nucleus was devoid of DNA labelling (Fig. 1e; far right).

\section{Mitochondrial membrane potential}

In this study, it is possible to recognise three levels of MMP (Fig. 4). High MMP was characterised by the majority of the mitochondria in the midpiece of the spermatozoon showing evidence of red/orange fluorescence (Fig. 4a and d); mitochondria of low MMP were characterised by a green fluorescence (Fig. $4 \mathrm{~b}$ and e), while in other rare occasions, an absence of MMP (Fig. 4c and f) was also noted. Thus, MMP could be co-localised with the membrane viability, producing a total of six different classes of spermatozoa as shown in Fig. 4. Interestingly, a small fraction of spermatozoa that showed evidence of damaged plasma membranes (propidium iodide (PI)-positive sperm head) was still capable of possessing high or low MMP (Fig. 4d and e). Subsequent experiments in this study have only reported the effect of anisotonic excursions and cryopreservation on the percentage of spermatozoa with high MMP.

\section{The effect of anisotonic media on sperm integrity}

The effects of initial exposure on koala sperm plasma membrane integrity (PMI) MMP, chromatin relaxation and $\%$ SDF to a range of anisotonic media $(64,170$, 350, 680 and $1330 \mathrm{mOsm} / \mathrm{kg}$ ) (E) are shown in Fig. 5a, $\mathrm{b}, \mathrm{c}$ and $\mathrm{d}$. Figure 5 also illustrates the subsequent change in sperm integrity following processing of the diluted sperm suspension and its return to isotonic conditions (350 mOsm/ $\mathrm{kg}$ ) (R). Figure 5 a shows that the sperm plasma membrane was significantly damaged when spermatozoa were initially exposed to extreme hypotonic (64 and $170 \mathrm{mOsm} / \mathrm{kg}$ ) and hypertonic $(1332 \mathrm{mOsm} / \mathrm{kg})$ media. Interestingly, there was no further statistically significant detrimental change in membrane integrity once the spermatozoa were returned to the $350 \mathrm{mOsm} / \mathrm{kg}$ Tris-citrate-glucose (TCG) buffer. Figure $5 \mathrm{~b}$ indicates that high MMP declined in hypotonic (64 and $170 \mathrm{mOsm} / \mathrm{kg}$ ) and extreme hypertonic $(1330 \mathrm{mOsm} / \mathrm{kg}$ ) media, but the loss of MMP in the $64 \mathrm{mOsm} / \mathrm{kg}$ medium was greater than that observed in the $1332 \mathrm{mOsm} / \mathrm{kg}$ medium. On return of spermatozoa to the isotonic media, spermatozoa initially diluted in the $64 \mathrm{mOsm} / \mathrm{kg}$ medium showed a trend towards a significant increase in MMP $(P=0.08)$, while the MMP of spermatozoa initially diluted in $1330 \mathrm{mOsm} / \mathrm{kg}$, and then returned to isotonic conditions, was not significantly different from that of the initial sample. Figure 5c indicates how initial exposure of koala spermatozoa to the $64 \mathrm{mOsm} / \mathrm{kg}$ medium resulted in a significant increase in spermatozoa with relaxed chromatin; on the return of these spermatozoa to isotonic conditions, there was a further increase in spermatozoa with 

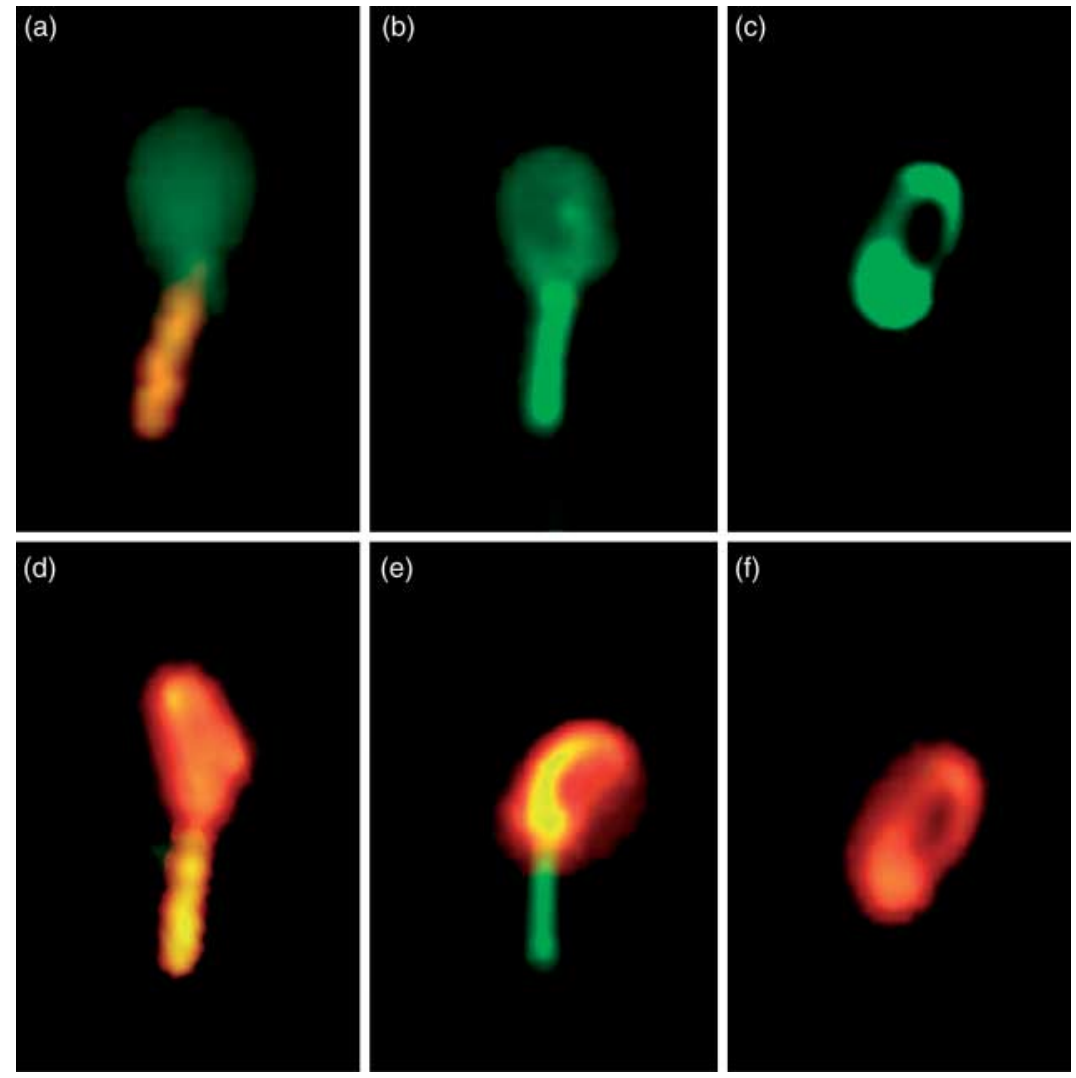

Figure 4 (a) Intact plasma membrane with high mitochondrial membrane potential (MMP);

(b) intact plasma membrane with low MMP;

(c) intact plasma membrane with no MMP;

(d) damaged plasma membrane with high MMP;

(e) damaged plasma membrane with low MMP and $(\mathrm{f})$ damaged plasma membrane with no MMP. relaxed chromatin. Spermatozoa initially diluted in the $170 \mathrm{mOsm} / \mathrm{kg}$ media showed no evidence of increased relaxed chromatin, but when these spermatozoa returned to the isotonic conditions, there was a significant increase in this parameter when compared with those found in the initial diluted sample. Figure $5 d$ also shows how initial exposure of koala spermatozoa to the 64,170 and $1330 \mathrm{mOsm} / \mathrm{kg}$ media resulted in significant increases in the percentage of spermatozoa with fragmented DNA when compared with the initial sample. Exposure of spermatozoa to the $64 \mathrm{mOsm} / \mathrm{kg}$ medium resulted in the highest level of SDF, and the subsequent return of these spermatozoa to isotonic conditions resulted in a further significant increase in \%SDF.

\section{Comparative effects of anisotonic media and cryopreservation on sperm integrity}

When compared with the sperm integrity of the initial sample, the cryopreservation procedure significantly reduced $\mathrm{PMI}$ and MMP but increased the percentage of spermatozoa with relaxed chromatin and damaged DNA (Fig. 5a, b, c and d). The proportion of spermatozoa with intact plasma membranes 30 min after cryopreservation was not significantly different from those exposed to the 64 or $170 \mathrm{mOsm} / \mathrm{kg}$ media or exposed and then returned to the isotonic media after exposure to the
$1330 \mathrm{mOsm} / \mathrm{kg}$ medium. The PMl of thawed spermatozoa was lower than that of spermatozoa exposed to the 350 and $680 \mathrm{mOsm} / \mathrm{kg}$ media and of spermatozoa following their initial exposure to the $1330 \mathrm{mOsm} / \mathrm{kg}$ media. The MMP of thawed spermatozoa was significantly higher than the spermatozoa initially exposed to the $64 \mathrm{mOsm} / \mathrm{kg}$ media and lower than that of spermatozoa initially exposed to the $350 \mathrm{mOsm} / \mathrm{kg}$ media; there were no other statistical differences noted between the thawed spermatozoa and sperm diluted in any other of the anisotonic diluents. The thawed spermatozoa, along with the spermatozoa exposed to the 64 and $170 \mathrm{mOsm} / \mathrm{kg}$ diluents, showed higher levels of relaxed chromatin compared with those in other anisotonic media. The highest proportion of spermatozoa with SDF was found in the semen samples exposed to the $64 \mathrm{mOsm} / \mathrm{kg}$ media; the proportion of sperm with SDF in this diluent was higher than that found in the thawed semen sample.

\section{Relationship between relaxed chromatin and percentage of SDF}

Figure 6A and B report the Pearson's $r$ correlation analyses of \%SDF and \% relaxed chromatin between two aliquots of spermatozoa from the same koala $(n=5)$ that were, respectively, cryopreserved and exposed to a $64 \mathrm{mOsm} / \mathrm{kg}$ hypotonic excursion. Figure $6 \mathrm{C}$ and D show the respective Pearson's $r$ correlation analyses 
A

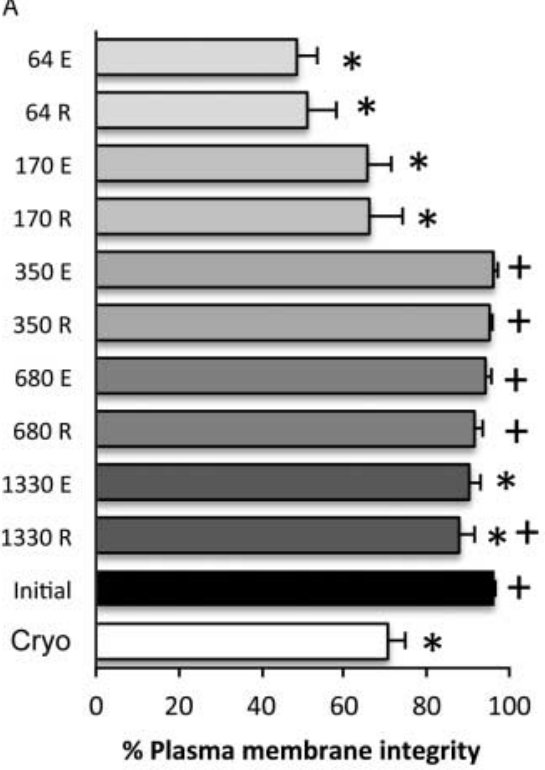

C

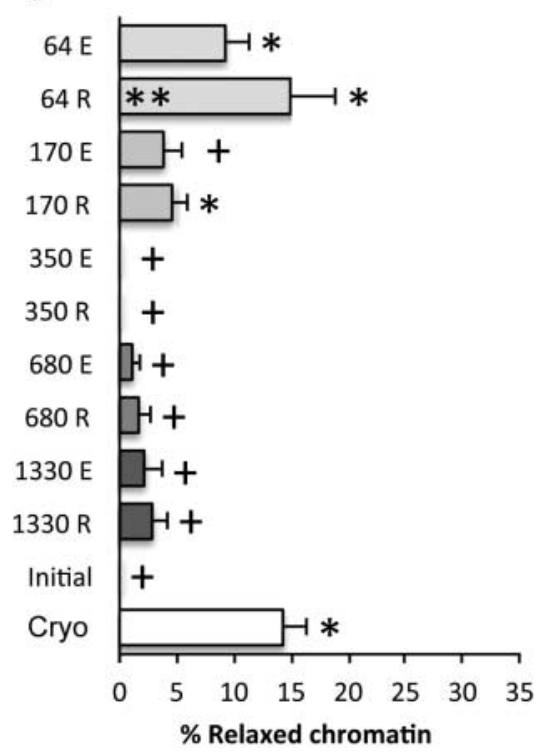

B

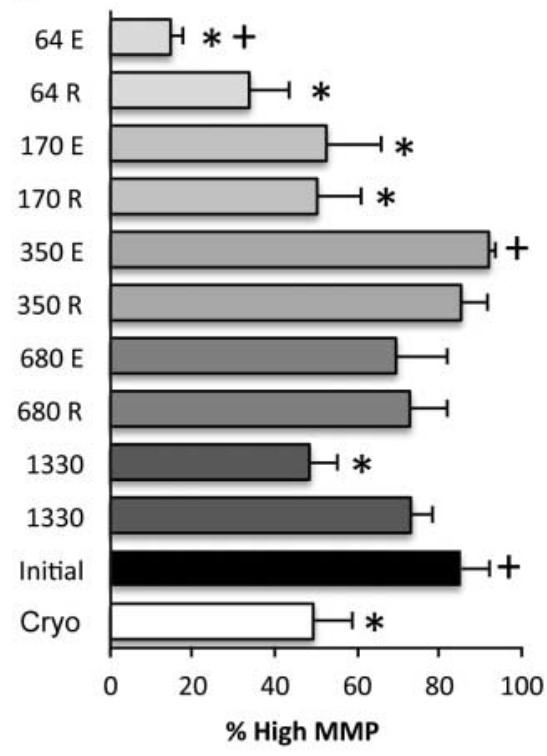

D

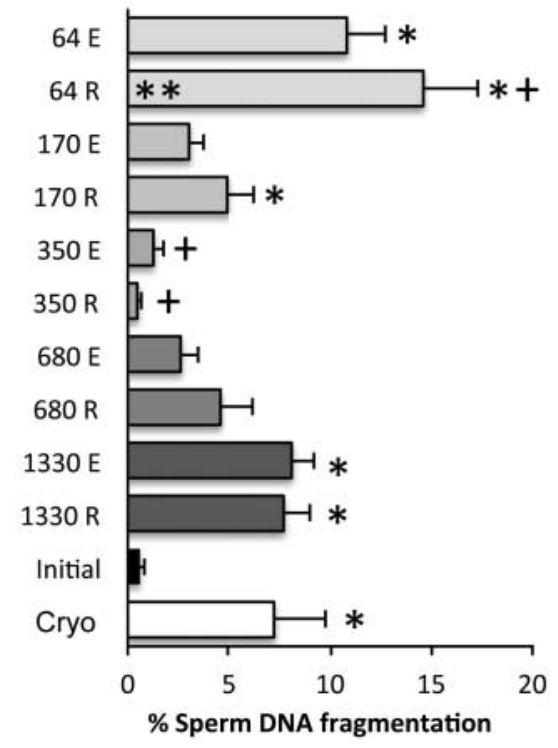

Figure 5 The effect of anisotonic media and cryopreservation (Cryo: post-thaw not washed) on the percentage of spermatozoa with $(\mathrm{A})$ intact plasma membranes (PMI), (B) high mitochondrial membrane potential (HMMP), (C) relaxed chromatin and (D) sperm DNA fragmentation (SDF). Key: E - initial osmotic excursion; $\mathrm{R}$ - return osmotic excursion back to an isotonic environment; * statistical difference $(P<0.05)$ when compared with the initial sample; ${ }^{* *}$ statistical difference between $\mathrm{E}$ and $\mathrm{R}$ samples of the same osmolality; + statistical difference $(P<0.05)$ when compared with the post-thaw semen sample Cryo. between \%SDF and \% relaxed chromatin of sperm following cryopreservation and after a $64 \mathrm{mOsm} / \mathrm{kg}$ hypotonic excursion. Figure $6 \mathrm{~A}$ and $\mathrm{B}$ show that exposure of koala sperm DNA to an extreme hypotonic excursion may be a potential model for explaining SDFinduced cryopreservation damage $(r=0.878 ; P=0.049)$, but it does not appear to be an explanation for relaxed chromatin $(r=-0.350 ; P=0.564)$. Figure $6 C$ and $D$ fail to support a strong relationship between relaxed chromatin and SDF.

\section{Discussion}

One of the primary objectives of this study was to examine whether pathology associated with osmotic flux of the non-frozen koala sperm could help better explain cryoinjury. The impact of osmotic changes on ejaculates, which are ex vivo handled, has been examined in a range of species, with particular attention to the effects of anisotonic media on the sperm plasma membrane and motility (Willoughby et al. 1996, Meyers 2005, Johnston et al. 2006), but this is the first time that this experimental approach has been used to examine the effect on SDF. An important feature of our experimental design was that spermatozoa extended from the same ejaculate were concurrently examined following exposure to anisotonic media and cryopreservation so that these effects could be directly compared. Results from this study have shown that the koala spermatozoa exposed to extreme anisotonic environments show a loss of membrane integrity and MMP and an overall increase in chromatin relaxation and SDF. The extent of these effects was 

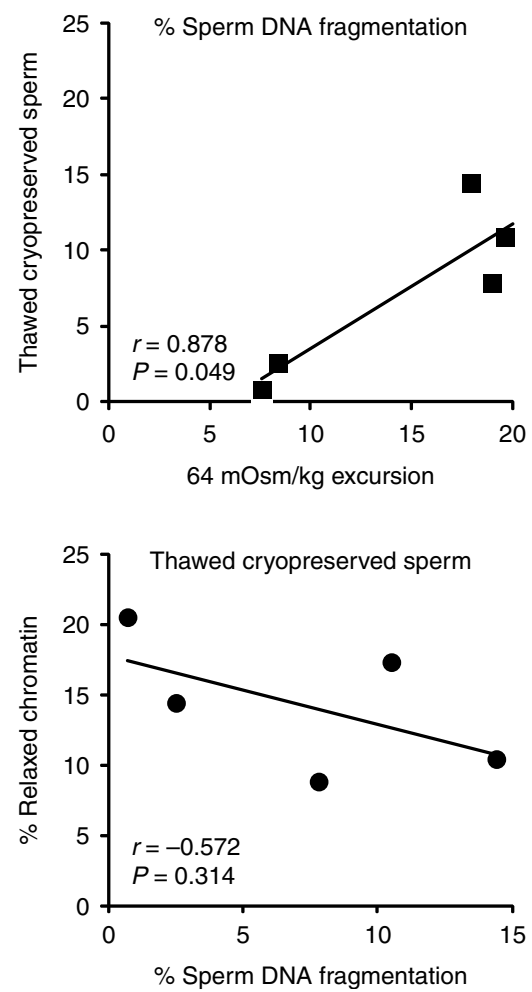
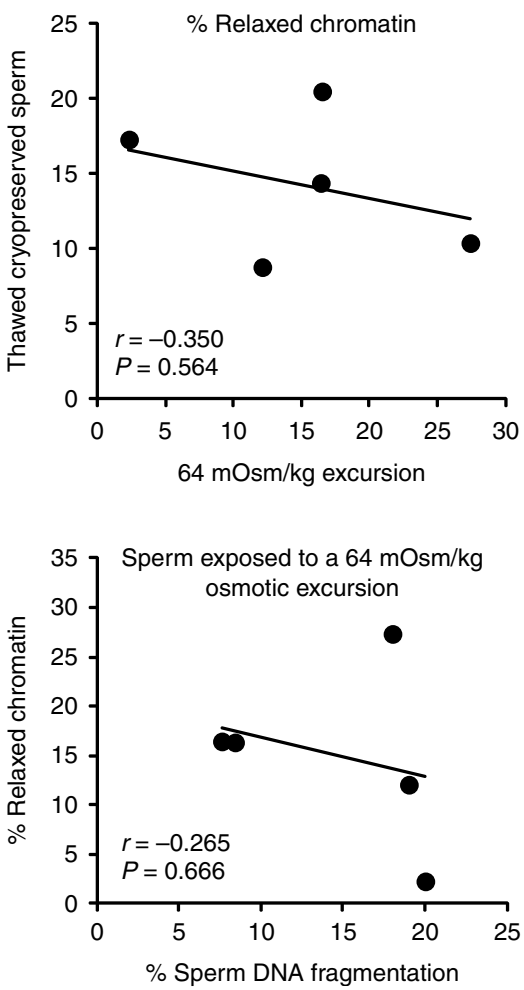

Figure 6 Pearson's $r$ correlations comparing the percentage of sperm with SDF and relaxed chromatin following cryopreservation and exposure to a $64 \mathrm{mOsm} / \mathrm{kg}$ osmotic excursion. (A) correlation of the \% of sperm with fragmented DNA following cryopreservation and induced osmotic damage; (B) correlation of the $\%$ of sperm with relaxed chromatin following cryopreservation and induced osmotic damage; (C) correlation of the percentage of sperm with relaxed chromatin and \%SDF following cryopreservation and (D) correlation of the percentage of sperm with relaxed chromatin and \%SDF following exposure to a $64 \mathrm{mOsm} / \mathrm{kg}$ excursion. dependent on the intensity of the osmotic stress, but exposure to hypotonic environments was particularly harmful to sperm integrity, except that of MMP which showed some degree of recovery when returned to an isotonic environment. The response of sperm integrity to hypotonic conditions essentially mimicked that described for spermatozoa following cryopreservation. These results are consistent with other studies which show that the osmotic stress associated with thawing is likely to be most detrimental to the spermatozoa during the cryopreservation procedure (Holt et al. 1992). The post-thaw membrane integrity reported in this study was higher than that reported in other studies on koala sperm examined immediately after cryopreservation (Johnston et al. 2006, Zee et al. 2008, 2009a) and reflects the fact that spermatozoa in the current study were examined 30 min after thawing; however, it should be noted that these studies have also reported a rapid decline in PMI of these same spermatozoa after $120 \mathrm{~min}$ of incubation at $35^{\circ} \mathrm{C}$. These observations draw attention to the importance of induced damage associated with ex vivo handling (so-called 'iatrogenic damage') and the variable survival time of spermatozoa when incubated within in vitro environments that attempt to mimic the physiology of the female reproductive tract. Additionally, it also reinforces the idea of using the spermatozoa as soon as possible for assisted reproductive technology, once the thawed samples have been processed.

The effect of osmosis on MMP is particularly interesting and suggests that koala sperm mitochondria are sensitive to hypotonic environments; clearly, changes in water volume of the koala spermatozoon during osmosis interfere with the physiological mechanisms that generate and maintain membrane potential. Spermatozoa exposed to hypertonic environments also showed a reduction in MMP, but this effect could be reversed if the spermatozoa were re-equilibrated back into an isotonic environment. This observation suggests that the physiology that drives mitochondrial function is capable of tolerating hypertonic environments better than they can cope with hypotonic changes. Interestingly, the percentage of koala spermatozoa with high MMP following cryopreservation (this study; Zee et al. 2007, 2009a) was similar to that of spermatozoa exposed to hypotonic conditions and, therefore, suggests that the thawing component of cryopreservation may be most detrimental to koala sperm mitochondria.

Koala spermatozoa showed a significant increase in chromatin relaxation when exposed to hypotonic conditions and following cryopreservation. On the face of it, these observations appear similar and suggest that chromatin relaxation is associated with an increase in the structural damage connected with changes in water flux across the plasma membrane. However, subsequent analysis of the data (Fig. 6) revealed no significant correlation between the incidence of spermatozoa with relaxed chromatin following cryopreservation and exposure to a $64 \mathrm{mOsm} / \mathrm{kg}$ osmotic excursion. It appears, therefore, that the respective causes of an 
increased incidence in relaxed chromatin following koala sperm cryopreservation and osmotic injury are not directly related to SDF, or more likely occur asynchronously. We offer at least two possible explanations to account for this phenomenon: 1 ) the nuclear membrane is somehow physically attached to the chromatin - then it is possible that water flux into the nucleus and/or general ice crystal damage to the sperm cell could result in a subsequent physical 'tearing' of the tertiary structure of the DNA-protamine complex, leading to an increase in the incidence of relaxed chromatin, and 2) the massive presence of alkali labile sites in koala sperm DNA, together with the absence of disulphide bonding to cross link protamines, predisposes the chromatin to relax, probably associated with the rapid production of single-stranded DNA motifs emerging from unpaired single-stranded stretches of either apurinic or apyrimidinic sites. The synergistic coexistence of both processes cannot be discarded. With respect to the first explanation, the existence of a physical connection between chromatin and the nuclear membrane is not well established, but there is evidence in human spermatozoa of a telomere-binding protein complex, identified as hSTBP (a variant of the $\mathrm{H} 2 \mathrm{~B}$ histone) which has a possible role in membrane attachment when associated with the telomeres (Gineitis et al. 2000). A possible relationship between sperm chromatin and the nuclear membrane could also involve certain lipophilic structures such as those described in sea urchin sperm nuclei, which together may function as pronuclear envelope organising centres (Collas \& Poccia 1995). Interestingly, these lipophilic structures are located at the acrosomal and centriolar poles of sea urchin sperm and initiate ATP-dependent vesicle binding in fertilised egg extracts. In some sense, these same sperm domains have also been identified in koalas as 'hot spots' to initiate sperm DNA damage. Breed et al. (2001) have noted the presence of a thin segment of the koala acrosome that runs from the main body of the acrosome towards the attachment of the tail and also laterally around the edges of the nucleus; again these areas are consistent with the DNA fragmentation 'hot spots' identified in the current study. Whatever the mechanism of chromatin relaxation, the koala sperm must have its own peculiar chromatin organisation, because relaxation of the chromatin in this species is not as pronounced as in the closely related wombat. Johnston et al. (2006) have shown that sperm chromatin relaxation in the wombat, following post-thaw incubation, is significantly lower than that found in the koala if the spermatozoa are frozen under the same conditions. Similarly, Breed et al. (2001) have also noted that the koala sperm nucleus is more prone to dispersion with Triton X-100 detergent than wombat spermatozoa, and this may be related to the presence of a large nuclear vacuole in the base of the koala sperm nucleus, which is not apparent in wombat. With respect to the second possibility, we have previously shown and proposed the notion that alkali-labile sites (ALS) present on the DNA (Zee et al. 2009b) facilitate the DNA bending necessary to configure the tertiary and ultimate structure of the DNA-protamine complex (Johnston et al. 2007). These ALS could be present in the mature spermatozoa in the form of unpaired single-stranded DNA stretches or simply as apurinic or apyrimidinic sites. This suggestion was made on the basis of the prominent presence of alkaline comets in normal spermatozoa (Zee et al. 2009b). It is possible that further structural damage and/ or oxidative attack to these potentially weakened areas may lead to chromatin relaxation. In addition, we cannot rule out the formation of newly formed single-stranded breaks leading to chromatin relaxation arising directly from osmotic membrane rupture (plasma membrane and mitochondria) and subsequent oxidative damage. The mechanism that leads to increased DNA breaks following exposure to hypotonic media requires further investigation but is likely to be similar to that resulting from cryoinjury. This was supported by the observation of a significant correlation of SDF between thawed cryopreserved spermatozoa and those exposed to a $64 \mathrm{mOsm} / \mathrm{kg}$ excursion $(r=0.878 ; P<0.05)$.

The other issue of interest emerging from our results is that DNA damage associated with cryoinjury occurred in a non-randomised manner. Thawing of koala spermatozoa results in the production of highly localised DNA damage that then extends to other chromosomal domains. ISNT revealed that the chromatin is first affected in the peri-acrosomal and basal regions. DNA labelling then subsequently expanded to other regions of the nucleus. It is also possible that peripheral DNA damage observed in this study, after loss of membrane stability and chromatin relaxation, is due to the presence of non-orthodox DNA conformations such as putative unpaired single-stranded DNA motifs or even abasic sites affecting one of the DNA strands. The presence of single-stranded DNA breaks in the periphery of the spermatozoa may act as putative DNA nicks that facilitate the production of further single-stranded DNA motifs and ultimately manifest as double-stranded breaks in highly affected spermatozoa, such as those presenting in this study as massive haloes of chromatin dispersion of the microgel following the SCD test. While this concept has not been thoroughly investigated, there are some observations where the sperm nuclei may exhibit discrete and preferable peripheral distribution of foci containing single-stranded DNA motifs and this fact has been linked to infertility in men (Zhang et al. 2007). A highly ordered form of DNA damage has also been reported in short-beaked echidna (Johnston et al. 2009). In this case, the combined use of ISNT and alkaline comets revealed the presence of a directional DNA nicking co-localised with the presence of highly sensitive ALS (putative single-stranded DNA motif generators after alkaline DNA denaturation) along the 
length of the sperm nucleus. While there was evidence of DNA damage starting from the base of the koala spermatozoa in this study, there was also apparent DNA damage adjacent to the acrosome; no such observations were made in echidna spermatozoa. The release of chromatin damaging agents such as proteases might be expected from the acrosome, particularly when associated with damaged plasma membranes. Although the marsupial acrosome is generally thought to be resistant to cryoinjury (Sistina et al. 1993, Breed et al. 2001, Johnston \& Holt 2001), this might still be a plausible explanation for co-localisation of initial DNA damage and the peri-acrosomal region in this study. Although the marsupial acrosome is known to be highly stable, even to the repeated effects of a freeze-thaw procedure (Sistina et al. 1993), rupture or more subtle damage of the underlying nuclear or acrosomal membranes might still occur in the peri-acrosomal region during cryopreservation. These events could plausibly induce digestion of proteins associated with these chromatin domains and may then lead to secondary structural changes in the adjacent DNA. The other region where DNA may be directly attacked due to the presence of localised oxidative stress is in close proximity to the mitochondria within the proximal midpiece at the base of the sperm head. The combined loss of plasma membrane function and damaged mitochondria following cryopreservation could be contributing to an elevated REDOX environment that is detrimental to sperm DNA.

On summarising, we propose an integrative model of cryo-induced osmotic injury for koala sperm that initially involves damage to membranes, which in combination with oxidative stress, leads to reduction of MMP and relaxation of chromatin in the short term followed by non-randomised DNA fragmentation.

\section{Materials and Methods}

\section{Animals and semen collection}

A total of seven sexually mature, clinically healthy captive male koalas were used to conduct this study. All animals and experiments were approved and conducted under permit SAS/297/08/UQFRG of the University of Queensland Animal Ethics Committee. Anaesthesia and the electroejaculation procedure in the koala have previously been described (Johnston et al. 1994, McGowan et al. 1995).

\section{Sperm preparation}

TCG buffer was used as the base diluent in this study and was composed of $3.0 \mathrm{~g}$ Tris base (Sigma-Aldrich), $1.7 \mathrm{~g}$ citric acid (Ajax Finechem, Taren Point, NSW, Australia), $1.25 \mathrm{~g}$ glucose (Ajax Finechem) made up to $100 \mathrm{ml}$ with Milli-Q Ultrapure water (Millipore Australia Pty Ltd., North Ryde, NSW, Australia) and adjusted to a $\mathrm{pH}$ of 7.4 and an osmolality of $350 \mathrm{mOsm} / \mathrm{kg}$ (Johnston et al. 2006). The 'control', a $350 \mathrm{mOsm} / \mathrm{kg}$ TCG buffer, was based on the osmolality of undiluted koala semen. Hypertonic solutions used in this study were prepared by adjustment of osmolality by addition of sucrose (BDH Chemicals Australia Pty Ltd., Melbourne, Vic, Australia) to the TCG buffer (680 and $1330 \mathrm{mOsm} / \mathrm{kg}$ ), whereas hypotonic solutions were prepared by dilution of TCG buffer with Milli-Q Ultrapure water (64 and $170 \mathrm{mOsm} / \mathrm{kg}$ ). TCG buffer with $28 \%(\mathrm{v} / \mathrm{v})$ glycerol was prepared as the cryopreservation medium and had a final working glycerol concentration of $14 \%(\mathrm{v} / \mathrm{v})$ (Johnston et al. 2006).

Koala semen cryopreservation has been described previously (Johnston et al. 2006, Zee et al. 2008). Briefly, each ejaculate was initially diluted $(1: 1)$ with warm $\left(35^{\circ} \mathrm{C}\right.$ - normal koala body temperature) TCG buffer in a pre-warmed $1.5 \mathrm{ml}$ microcentrifuge tube (Eppendorf AG, Hamburg, Germany) and allowed to cool to room temperature $\left(25^{\circ} \mathrm{C}\right)$ over a period of $10 \mathrm{~min}$. The extended semen sample was placed into a $10 \mathrm{ml}$ flask containing $\sim 2 \mathrm{ml}$ of water at $25^{\circ} \mathrm{C}$ and subsequently placed into a refrigerator to equilibrate to $4{ }^{\circ} \mathrm{C}$ over $\sim 2 \mathrm{~h}$. This chilled, equilibrated sample was assessed as the initial (T0) sample. The semen sample was then analysed for PMI, MMP, chromatin relaxation and SDF. Chilled $\left(4^{\circ} \mathrm{C}\right)$ cryopreservation media was added, drop-wise, to the extended chilledequilibrated semen. Semen samples were drawn into prechilled $0.25 \mathrm{ml}$ straws (IMV, L'Aigle, France), sealed and frozen in a programmable freezer at $6^{\circ} \mathrm{C} / \mathrm{min}$ (Freeze Control CL-863, Cryologics Pty Ltd., Mulgrave, Vic, Australia) from $4{ }^{\circ} \mathrm{C}$ to $-80^{\circ} \mathrm{C}$. Straws were then removed from the freezer, plunged into liquid nitrogen $\left(\mathrm{LN} ;-196^{\circ} \mathrm{C}\right)$ and stored. Straws were thawed in a $35^{\circ} \mathrm{C}$ water bath for $1 \mathrm{~min}$ and semen samples then dispensed into pre-warmed $1.5 \mathrm{ml}$ microcentrifuge tubes.

\section{Assessment of semen quality}

PMI of the spermatozoa was determined using a dual fluorescent staining procedure; $100 \mathrm{nM}$ SYBR-14 and $12 \mu \mathrm{M}$ PI from the Live/DeadSperm viability kit (L-7011; Invitrogen Australia Pty Limited) was used according to the manufacturer's instructions (Zee et al. 2007). MMP was determined using a lipophilic cationic fluorescent probe 5,5',6,6' $6^{\prime}$-tetrachloro$1,1^{\prime}, 3,3^{\prime}$-tetraethylbenzimidazolyl carbocyanine iodide (JC-1; Invitrogen Australia Pty Ltd.) that has an excitation range of 485 to $535 \mathrm{~nm}$ (J-aggregates only). Each sample was stained with final concentration of $2 \mu \mathrm{M} \mathrm{JC}-1$, counterstained using the PMI technique above and incubated for $30 \mathrm{~min}$. The JC-1 probe indicated a potential accumulation in the mitochondria where the monomeric molecules (emission at $530 \mathrm{~nm}$ ) accumulate to form J-aggregates with increasing mitochondrial potential (emission at $590 \mathrm{~nm}$ ). Spermatozoa were categorised into three levels of mitochondrial potential: none, low (LMMP) and high (HMMP) with no fluorescence, green fluorescence and orange fluorescence respectively. Fluorescence was assessed using an epifluorescence microscope (Nikon Eclipse E400; FITC 465-495 excitation filter; DM 505 dichroic mirror; BA 515-555 barrier filter).

SDF was determined using a Halomax kit (Halotech SL, Madrid, Spain) previously validated and described for koala spermatozoa first by Johnston et al. (2007) and then refined by Zee et al. (2009b). ISNT of DNA breaks was determined on cryopreserved semen samples $(n=2)$. Samples were incubated 
for $2 \mathrm{~h}$ at $35^{\circ} \mathrm{C}$ post-thaw. ISNT was conducted on samples with and without protein lysis. Spermatozoa were mixed into low-density agarose, and microgels were prepared onto glass slides, then dehydrated in a series of increasing ethanol solutions (70, 90 and 100\%), dried for $5 \mathrm{~min}$, and the glass coverslip was removed before ISNT. Reaction buffer containing 5 units of DNA polymerase I endonuclease free (Kornberg polymerase; Roche Diagnostics $\mathrm{GmbH}$ ) and $10 \mu \mathrm{M}$ digoxigenin-11-dUTP (Roche Diagnostics $\mathrm{GmbH}$ ) was dispensed onto the microgel, covered with a plastic coverslip and incubated in a humidified chamber for 5, 10, 20 and $30 \mathrm{~min}$ at $37^{\circ} \mathrm{C}$. Slides were washed in Tris-borate-EDTA buffer $(\mathrm{pH}$ 8), dehydrated in sequential ethanol solutions (70, 90 and 100\%) and air-dried. Digoxigenin-11-dUTP was detected using anti-digoxigenin-fluorescein, Fab Fragments (Roche Diagnostics $\mathrm{GmbH}$ ) incubated for $30 \mathrm{~min}$. The slides were counterstained with PI $(2 \mu \mathrm{g} / \mathrm{ml})$ in Vectashield (H 1100, Vector, Burlingame, CA, USA). In order to control for background binding, designated areas of the microgels were incubated with the reaction buffer alone, omitting the DNA polymerase I, and a small section of the microgels between those areas was removed to avoid diffusion of the enzyme. Following ISNT, spermatozoa within the microgels were imaged at $\times 600$ magnification, using Leica DMRB microscope (Leica Microsystems, Germany) to determine degrees of chromatin relaxation and the proportion of the nucleus positively stained with the DNA label. The ISNT-unlabelled spermatozoa typically stained red if the nucleotides were not incorporated but exhibited green fluorescence if traces of DNA damage were present. The area of the nucleus represented by the red fluorescence was then correlated with the integrated density of the green DNA label. Fluorescent images were captured as.tiff 12-bit files using a black and white cooled Leica DCF 300 camera with single band pass filters (FITC-3540B-536/617; TxRed-4040C-000; Semrock, Rochester, NY, USA). Image analysis was performed using Leica Q-Win software (LeicaMicrosystems, Barcelona, Spain). Colour coding was selected to improve visual discrimination between both fluorescent channels. Images were transformed into colour by converting. tiff 12-bit images into.tiff 8-bit images and colour assignment, $\mathrm{red} / \mathrm{blue}$ for DNA and the green for proteins, to the 8-bit grey level images using Adobe Photoshop 7.0 (Adobe Systems Incorporated).

\section{The effect of anisotonic media on sperm integrity}

This study examined the potential role of osmotic flux associated with the loss of koala sperm integrity following cryopreservation. Initial assessments were carried out on ejaculated semen samples $(n=5)$ that were diluted $1: 1$ with TCG and stored chilled $\left(4^{\circ} \mathrm{C}\right)$ before use. These samples were then equilibrated at $25^{\circ} \mathrm{C}$ for $5 \mathrm{~min}$ and incubated for a further 10 min at $35^{\circ} \mathrm{C}$; subsamples were stained for assessment of PMI, MMP and chromatin integrity. A $10 \mu \mathrm{l}$ aliquot of the initial semen sample was then extended into $190 \mu \mathrm{l}$ of the anisotonic test media and incubated at $35^{\circ} \mathrm{C}$ for $10 \mathrm{~min}$ (sufficient time to induce osmotic stress (Johnston et al. 2006)). This exposure represented the first or initial osmotic excursion (E); subsamples were stained for assessment of PMI, MMP and chromatin integrity. The remaining semen sample was washed by centrifugation at $200 \mathrm{~g}$ for $30 \mathrm{~s}$, the supernatant was removed and the pellet was resuspended in TCG medium $\left(350 \mathrm{mOsm} / \mathrm{kg}\right.$ ) and incubated at $35^{\circ} \mathrm{C}$ for $10 \mathrm{~min}$. This exposure represented the second osmotic excursion and a return to an isotonic environment $(R)$; subsamples were stained for assessment of PMI, MMP and chromatin integrity.

\section{Effect of post-thaw dilution on sperm integrity}

Concurrent to the aforementioned exposure of spermatozoa to varying osmotic stress, an aliquot of the initial sample was cryopreserved and thawed in order to examine post-thaw PMI, MMP and chromatin integrity in order to compare the results with non-frozen spermatozoa exposed to the anisotonic media. Two frozen semen straws were thawed and pooled and incubated with no further processing so that spermatozoa were left in $14 \%$ glycerol v/v. All cryopreserved koala sperm samples were assessed for PMI, MMP and chromatin integrity after $10 \mathrm{~min}$ of incubation at $35^{\circ} \mathrm{C}$ within 30 min of thawing.

\section{Statistical analysis}

Statistical analysis was performed using the Statistical Package for the Social Sciences 17 (SPSS, Inc., Chicago, IL, USA). All percentage data were arcsine transformed before analysis. Non-parametric statistics for a non-Gaussian population including pairwise comparisons using exact one-sided Wilcoxon tests were performed. Correlation analysis was conducted using a Person's $r$ test. Image analysis was performed to compare the fluorescence intensities obtained after ISNT and the area of the spermatozoa (measured in pixels) as an index of chromatin relaxation. The analysis was performed on 50 different sperm nuclei with protein depletion exhibiting different levels of chromatin relaxation and DNA labelling. For this purpose, integrated density (a correlation between the area of interest and fluorescence intensity after background subtraction) was calculated for analysis of fluorescence intensity. Areas of the nuclei were represented as the number of pixels after using automatic image analysis threshold algorithms based on background analysis against the regions of interest. Leica Q-Win image analysis software (LeicaMicrosystems Germany) was used.

\section{Declaration of interest}

The authors declare that there is no conflict of interest that could be perceived as prejudicing the impartiality of the research reported.

\section{Funding}

This project was supported by an Australian Research Council Linkage Grant (LP0455785) and the Spanish Ministry of Science and Technology (MCYT: BFU2010-16738). 


\section{Acknowledgements}

The authors would like to thank the staff at Moggill Koala Hospital, Dreamworld and Currumbin Wildlife Sanctuary who contributed their time and expertise in koala handling, husbandry and anaesthesia. The authors would especially like to acknowledge Dr Allan McKinnon, Mr Peter Theilemann, Dr Vere Nicolson and Dr Michael Pyne.

\section{References}

Breed WG, Leigh CM \& Ricci M 2001 The structural organisation of sperm head components of the wombat and koala (suborder: Vombatiformes): an enigma amongst marsupials. Journal of Anatomy 198 57-66. (doi:10.1046/j.1469-7580.2001.19810057.x)

Collas P \& Poccia D 1995 Lipophilic organizing structures of sperm nuclei target membrane vesicle binding and are incorporated into the nuclear envelope. Developmental Biology 169 123-135. (doi:10.1006/dbio. 1995.1132)

Cummins JM 1980 Decondensation of sperm nuclei of Australian marsupials: effects of air drying and of calcium and magnesium. Gamete Research 3 351-367. (doi:10.1002/mrd.1120030407)

Curry MR \& Watson PF 1994 Osmotic effects on ram and human sperm membranes in relation to thawing injury. Cryobiology 31 39-46. (doi:10.1006/cryo.1994.1005)

Duchen MR 2004 Mitochondria in health and disease: perspectives on a new mitochondrial biology. Molecular Aspects of Medicine 25 365-451. (doi:10.1016/j.mam.2004.03.001)

Gineitis AA, Zalenskaya IR, Yau PM, Morton Bradbury E \& Zalensky AO 2000 Human sperm telomere-binding complex involves histone $\mathrm{H} 2 \mathrm{~B}$ and secures telomere membrane attachment. Journal of Cell Biology 151 1591-1597. (doi:10.1083/jcb.151.7.1591)

Holt WV 2000 Fundamental aspects of sperm cryobiology: the importance of species and individual differences. Theriogenology 53 47-58. (doi:10.1016/S0093-691X(99)00239-3)

Holt WV, Head MF \& North RD 1992 Freeze-induced membrane damage in ram spermatozoa is manifested after thawing - observations with experimental cryomicroscopy. Biology of Reproduction 46 1086-1094. (doi:10.1095/biolreprod46.6.1086)

Johnston SD \& Holt WV 2001 Germplasm conservation in marsupials. In Cryobanking the Genetic Resource. Wildlife Conservation for the Future? pp 201-27, ch 12. Eds PF Watson \& WV Holt. London: Taylor \& Francis.

Johnston SD, McGowan MR, Carrick FN, Cameron RDA \& Tribe A 1994 Seminal characteristics and spermatozoal morphology of captive Queensland koalas (Phascolarctos cinereus). Theriogenology 42 501-511. (doi:10.1016/0093-691X(94)90688-F)

Johnston SD, MacCallum C, Blyde D, McClean R, Lisle A \& Holt WV 2006 An investigation into the similarities and differences governing the cryopreservation success of koala (Phascolarctos cinereus: Goldfuss) and common wombat (Vombatus ursinus: Shaw) spermatozoa. Cryobiology 53 218-228. (doi:10.1016/j.cryobiol.2006.06.001)

Johnston SD, López-Fernández C, Gosálbez A, Zee Y, Holt WV, Allen C \& Gosálvez J 2007 The relationship between sperm morphology and chromatin integrity in the koala (Phascolarctos cinereus) as assessed by the sperm chromatin dispersion test (SCDt). Journal of Andrology 28 891-899. (doi:10.2164/jandrol.107.003350)
Johnston SD, López-Fernández C, Gosálbez A, Holt WV \& Gosálvez J 2009 Directional mapping of deoxyribonucleic acid nicking in ejaculated and cauda epididymidal spermatozoa of the short-beaked echidna (Tachyglossus aculeatus: Monotremata). Reproduction, Fertility, and Development 21 1008-1014. (doi:10.1071/RD09079)

McGowan MR, Tribe A, Johnston SD, Carrick FN \& Hensbroek L 1995 The use of isoflurane to anaesthetise koalas (Phascolarctos cinereus). Australian Veterinary Journal 72 472-473. (doi:10.1111/j.1751-0813. 1995.tb03495.x)

Meyers SA 2005 Spermatozoal response to osmotic stress. Animal Reproduction Science 89 57-64. (doi:10.1016/j.anireprosci.2005.06. 026)

Retief JD, Krajewski C, Westerman M, Winkfein RJ \& Dixon GH 1995 Molecular phylogeny and evolution of marsupial protamine P1 genes. Proceedings of the Royal Society of London 259 7-14. (doi:10.1098/ rspb.1995.0002)

Sistina Y, Lin M, Mate KE, Robinson ES \& Rodger JC 1993 The unique stability of the marsupial sperm acrosomal membranes examined by unprotected freeze-thawing and treatment with the detergent Triton X-100. Reproduction, Fertility, and Development 5 1-14. (doi:10.1071/ RD9930001)

Watson PF \& Fuller BJ 2001 Principles of cryopreservation of gametes and embryos. In Cryobanking the Genetic Resource: Wildlife Conservation for the Future? pp 23-46. Eds PF Watson \& WV Holt. London: Taylor \& Francis.

Willoughby CE, Mazur P, Peter AT \& Critser JK 1996 Osmotic tolerance limits and properties of murine spermatozoa. Biology of Reproduction $\mathbf{5 5}$ 715-727. (doi:10.1095/biolreprod55.3.715)

Zee Y, Holt WV, Allen CD, Nicolson V, Burridge M, Lisle A, Carrick FN \& Johnston SD 2007 Effects of cryopreservation on mitochondrial function and heterogeneity, lipid raft stability and phosphatidylserine translocation in koala (Phascolarctos cinereus) spermatozoa. Reproduction, Fertility, and Development 19 850-860. (doi:10.1071/RD07084)

Zee Y, Holt WV, Gosálvez J, Allen CD, Nicolson V, Pyne M, Burridge M, Carrick FN \& Johnston SD 2008 Dimethylacetamide can be used as an alternative to glycerol for the successful cryopreservation of koala (Phascolarctos cinereus) spermatozoa. Reproduction, Fertility, and Development 20 724-733. (doi:10.1071/RD08036)

Zee Y, Holt WV, Nicolson N, Pyne M \& Johnston SD 2009a Individual variability in post-thaw sperm survival in a captive koala population. Cryobiology 59 69-74. (doi:10.1016/j.cryobiol.2009.04.010)

Zee Y, López-Fernández C, Arroyo F, Johnston SD, Holt WV \& Gosálvez J $2009 b$ Evidence that single-stranded DNA breaks are a normal feature of koala sperm chromatin, while double-stranded DNA breaks are indicative of DNA damage. Reproduction 138 267-278. (doi:10.1530/ REP-09-0021)

Zhang X, San Gabriel M, Libman J, Phillips S, Courchesne A \& Zini A 2007 Localization of single-stranded DNA in human sperm nuclei. Fertility and Sterility 88 1334-1338. (doi:10.1016/j.fertnstert.2007.01.025)

Received 13 November 2011

First decision 2 February 2012

Revised manuscript received 5 April 2012

Accepted 12 April 2012 